\title{
Stability of low-dimensionally-confined bipolarons
}

\author{
R.T. Senger ${ }^{\mathrm{a}}$ and A. Erçelebi \\ Department of Physics, Bilkent University, 06533 Ankara, Turkey
}

Received 15 September 1999 and Received in final form 20 March 2000

\begin{abstract}
In the limit of strong electron-phonon coupling, we provide a unified insight into the stability criterion for bipolaron formation in low-dimensionally confined media. The model that we use consists of a pair of electrons immersed in a reservoir of bulk LO phonons and confined within an anisotropic parabolic potential box, whose barrier slopes can be tuned arbitrarily from zero to infinity. Thus, encompassing the bulk and all low-dimensional geometric configurations of general interest, we obtain an explicit tracking of the critical ratio $\eta_{\mathrm{c}}$ of dielectric constants below which bipolarons can exist.
\end{abstract}

PACS. 71.38.+i Polarons and electron-phonon interactions

\section{Introduction}

Depending on the relevant dielectric properties, two electrons in a polar medium can bind into a composite complex, termed a bipolaron. An enormous amount of literature published within this context leads to the evidence that bipolarons can exist under certain circumstances defined critically by the Coulomb repulsion coefficient and the electron-phonon coupling strength [1-16]. Of particular relevance to the content of the present article are the recent solutions of this problem in strict two dimensions (2D) $[6-9,12]$ where it has been found that bipolaron formation should be easier in a space of low dimensions. In this report we would like to extend the bipolaron problem into a more general context and present a comprehensive review of the two-polaron system in a confined medium through a description interpolating between integer-dimensional-space limits. We adopt an oversimplified model of a pair of electrons immersed in a reservoir of bulk LO phonons and confined within a deformable threedimensional box of parabolic boundary strengths, given in usual polaron units $\left(m^{\star}=\hbar=\omega_{\mathrm{LO}}=1\right)$ by

$$
V_{\text {conf }}(\varrho, z)=\frac{1}{2}\left(\Omega_{\varrho}^{2} \varrho^{2}+\Omega_{z}^{2} z^{2}\right)
$$

in which the dimensionless frequencies $\Omega_{\varrho}$ and $\Omega_{z}$ serve for the measures of the degree of confinement of the electrons in the respective lateral $(\varrho)$ and $\pm z$ directions. Setting $\Omega_{\varrho}=0$ and varying $\Omega_{z}$ from zero to infinity, one can trace the variational upper estimate for the bipolaron energy and the corresponding stability criterion interpolating between the three and two-dimensional space limits. On the other hand, deleting the confining potential along the $z$-axis $\left(\Omega_{z}=0\right)$ and fixing $\Omega_{\varrho}$ at non-zero finite values, the theory reflects the Q1D-description in

\footnotetext{
a e-mail: senger@fen.bilkent.edu.tr
}

a free standing QWW-like tubular structure. Hereafter, wherever relevant, we shall use $\Omega$ to mean $\Omega_{\varrho}\left(\Omega_{z}\right)$ when $\Omega_{z}\left(\Omega_{\varrho}\right)=0$. In the spherically symmetric box-type configuration we shall simply set $\Omega_{\varrho}=\Omega_{z}=\Omega$.

The above choice for the confining potential is appealing in the sense that the static depletion fields achieved in microstructures such as quantum wires and dots which are laterally confined by Schottky gates exhibit nearly parabolic potentials. Besides this, the usage of quadratic potential profiles greatly facilitates the calculations and leads to rather concise and tractable analytic expressions. We have thus refrained ourselves from treating potentials of other forms which possibly would lead to complicated and even prohibitively difficult expressions and numerical complications and yet yield qualitative features similar to those for parabolic potential shapes. Indeed, calculations pertaining to the cyclotron study of polarons confined to an interface indicate that the phonon-coupling - induced shift in the resonant energy is sensitive dominantly to the strength of the confining potential rather than its shape [17]. Moreover, due to the absence of an abrupt variation in the medium structure and properties, the parabolic confining potential allows one to disregard any pertinence to the interface phonon modes [18].

A complementary remark regarding the particular parabolic form of the potential used here is that it also finds its relevance in the study of bipolarons under external magnetic fields, where a field for a 3D-bipolaron conforms it effectively to a Q1D-bipolaron and a field applied normally to a Q2D-system makes it effectively zerodimensional. Specifically, under the symmetric gauge for the magnetic vector potential, one readily achieves the cyclotron frequency (measured in units of $\omega_{\mathrm{LO}}$ ) to undertake the role of the confining parameter $\Omega_{\varrho}$.

We should reemphasize that the fundamental approach followed in this work is to take into account solely the generic low dimensional aspect of the dynamical behavior 
of the confined electrons and visualize them as interacting with the medium and with one another through exchange of virtual phonons. Thus, adopting the so-called bulk-phonon approximation and leaving out all the other complicating detailed features, we study the ground state of confined bipolarons at strong phonon coupling and obtain an explicit tracking of the polaron-bipolaron transition as a function of the degree of confinement.

\section{Theory}

The Hamiltonian describing the confined electron pair system coupled to LO-phonons is given by

$$
\begin{aligned}
\mathbf{H}= & H_{\mathrm{e}}+\sum_{Q} a_{Q}^{\dagger} a_{Q}+\sum_{Q} V_{Q}\left[a _ { Q } \left\{\exp \left(\mathrm{i} \mathbf{Q} \cdot \mathbf{r}_{1}\right)\right.\right. \\
& \left.\left.+\exp \left(\mathrm{i} \mathbf{Q} \cdot \mathbf{r}_{2}\right)\right\}+\mathrm{hc}\right]
\end{aligned}
$$

where

$$
H_{\mathrm{e}}=\frac{1}{2} \sum_{i=1,2}\left(p_{i}^{2}+\Omega_{\varrho}^{2} \varrho_{i}^{2}+\Omega_{z}^{2} z_{i}^{2}\right)+\frac{U}{\left|\mathbf{r}_{1}-\mathbf{r}_{2}\right|} .
$$

In the above, $a_{Q}\left(a_{Q}^{\dagger}\right)$ is the phonon annihilation (creation) operator and $\mathbf{r}_{i}=\left(\boldsymbol{\rho}_{i}, z_{i}\right),(i=1,2)$, are the positions of the electrons in cylindrical coordinates. $p_{1}$ and $p_{2}$ denote the momenta of the electrons. The interaction amplitude is related to the electron-phonon coupling constant $\alpha$ and the phonon wavevector $\mathbf{Q}=\left(\mathbf{q}, q_{z}\right)$ through $V_{Q}=(2 \sqrt{2} \pi \alpha)^{1 / 2} / Q$. The coupling constant is given, in terms of the high frequency and static dielectric constants of the material, by

$$
\alpha=\frac{e^{2}}{\sqrt{2}}\left(\frac{1}{\epsilon_{\infty}}-\frac{1}{\epsilon_{0}}\right)
$$

in terms of which the unscreened Coulomb repulsive amplitude is

$$
U=\frac{e^{2}}{\epsilon_{\infty}}=\frac{\alpha \sqrt{2}}{1-\eta}, \quad \eta=\epsilon_{\infty} / \epsilon_{0}<1 .
$$

In the center of mass and relative coordinates:

$$
\mathbf{R}=\frac{1}{2}\left(\mathbf{r}_{1}+\mathbf{r}_{2}\right), \quad \mathbf{r}=\mathbf{r}_{1}-\mathbf{r}_{2}
$$

with corresponding momenta $\mathbf{P}=\mathbf{p}_{1}+\mathbf{p}_{2}$ and $\mathbf{p}=$ $\frac{1}{2}\left(\mathbf{p}_{1}-\mathbf{p}_{2}\right)$, the Hamiltonian can be rewritten as

$$
\begin{aligned}
\mathbf{H}= & H_{\mathrm{e}}+\sum_{Q} a_{Q}^{\dagger} a_{Q} \\
& +2 \sum_{Q} V_{Q} \cos \left(\frac{1}{2} \mathbf{Q} \cdot \mathbf{r}\right)\left[a_{Q} \mathrm{e}^{\mathbf{i} \mathbf{Q} \cdot \mathbf{R}}+\mathrm{hc}\right]
\end{aligned}
$$

where

$$
\begin{aligned}
H_{\mathrm{e}}= & \frac{1}{4} P^{2}+p^{2} \\
& +\Omega_{\varrho}^{2}\left(R_{\varrho}^{2}+\frac{1}{4} r_{\varrho}^{2}\right)+\Omega_{z}^{2}\left(R_{z}^{2}+\frac{1}{4} r_{z}^{2}\right)+\frac{U}{r}
\end{aligned}
$$

in which $\mathbf{R}_{\varrho}$ and $R_{z}$ stand for the lateral and $z$ components of the center of mass position vector. The components $\mathbf{r}_{\varrho}$ and $r_{z}$ have similar meanings for the relative position vector $\mathbf{r}$.

In the variational approximation that we follow here, we use the conventional trial Ansatz of the adiabatic polaron theory consisting of a part relevant to the phonon variables and a part which contains the particle coordinates only, i.e.,

$$
\Psi_{\mathrm{bipol}}=\Phi\left(\mathbf{r}_{1}, \mathbf{r}_{2}\right)|0\rangle
$$

where $|0\rangle$ denotes the phonon vacuum state. The bipolaron state, $\Psi_{\text {bipol }}$, thus constructed, is to be used in concert with the optimal displaced-oscillator transformation

$$
\mathrm{H} \rightarrow \mathcal{H}=\exp (-\mathcal{S}) \mathrm{H} \exp (\mathcal{S}), \quad \mathcal{S}=\sum_{Q} V_{Q} s_{Q}\left(a_{Q}-a_{Q}^{\dagger}\right)
$$

in which

$$
s_{Q}=\left\langle\Phi\left(\mathbf{r}_{1}, \mathbf{r}_{2}\right)\left|\left(\mathrm{e}^{ \pm \mathrm{i} \mathbf{Q} \cdot \mathbf{r}_{1}}+\mathrm{e}^{ \pm \mathrm{i} \mathbf{Q} \cdot \mathbf{r}_{2}}\right)\right| \Phi\left(\mathbf{r}_{1}, \mathbf{r}_{2}\right)\right\rangle .
$$

A further optimization of

$$
E_{\mathrm{g}} \equiv\left\langle\Psi_{\text {bipol }}|\mathcal{H}| \Psi_{\text {bipol }}\right\rangle
$$

with respect to the variational parameters contained in $\Phi\left(\mathbf{r}_{1}, \mathbf{r}_{2}\right)$ corresponds to the self-trapping description of the polarons where the charge density distribution of the electrons and the lattice polarization influence each other in such a way that a stable relaxed state of the "twoelectron + phonon" complex is eventually attained.

To reach this goal we need to use an appropriate form for $\Phi\left(\mathbf{r}_{1}, \mathbf{r}_{2}\right)$ that suits to reflect a reasonable description of the bipolaron state in a wide range of the confining parameters extending from the bulk to all low dimensional geometries. As a sensible approximation, we set

$$
\Phi\left(\mathbf{r}_{1}, \mathbf{r}_{2}\right) \propto G\left(\mathbf{r}_{1}\right) G\left(\mathbf{r}_{2}\right) g\left(r_{1}, r_{2},\left|\mathbf{r}_{1}-\mathbf{r}_{2}\right|\right)
$$

where $G\left(\mathbf{r}_{i}\right)(i=1,2)$ are the one-electron Gaussian wavefunctions, and $g$ is the Coulomb correlation function of Jastrow type. Compatible with the anisotropy imposed by the confining potential (1), we write

$$
G\left(\mathbf{r}_{i}\right)=\exp \left(-a_{1}^{2} \varrho_{i}^{2}-a_{2}^{2} z_{i}^{2}\right)
$$

and

$$
g\left(\mathbf{r}_{1}, \mathbf{r}_{2},\left|\mathbf{r}_{1}-\mathbf{r}_{2}\right|\right)=\sqrt{r_{\varrho}^{2}+r_{z}^{2}} \exp \left(b_{1}^{2} r_{\varrho}^{2}+b_{2}^{2} r_{z}^{2}\right)
$$

in which, the factor $r=\sqrt{r_{\varrho}^{2}+r_{z}^{2}}$ multiplying the exponential ensures that $\Phi(\mathbf{r}=0)=0$, so that the electrons are repulsively kept separated.

Under the coordinate transformations (5), it is possible to conform $\Phi$ into a product form $\Phi(\mathbf{R}, \mathbf{r})=\phi(\mathbf{R}) \times \varphi(\mathbf{r})$, 
where each of $\phi$ and $\varphi$ assumes an oscillator-type waveform, given by

$$
\begin{aligned}
\phi(\mathbf{R}) & =N_{R} \exp \left\{-\frac{1}{2} \kappa_{1}^{2}\left(R_{\varrho}^{2}+\mu_{1}^{2} R_{z}^{2}\right)\right\} \\
\varphi(\mathbf{r}) & =N_{r} \sqrt{r_{\varrho}^{2}+r_{z}^{2}} \exp \left\{-\frac{1}{2} \kappa_{2}^{2}\left(r_{\varrho}^{2}+\mu_{2}^{2} r_{z}^{2}\right)\right\}
\end{aligned}
$$

where the coefficients $N_{R}$ and $N_{r}$ serve for normalisation, and $\left\{\kappa_{i}, \mu_{i}\right\},(i=1,2)$ is the set of new variational parameters linked to those given in equations $(13,14)$ through

$$
\begin{aligned}
a_{1} & =\frac{1}{2} \kappa_{1}, \\
a_{2} & =\frac{1}{2} \kappa_{1} \mu_{1}, \\
b_{1}^{2} & =\frac{1}{8} \kappa_{1}^{2}-\frac{1}{2} \kappa_{2}^{2}, \\
b_{2}^{2} & =\frac{1}{8} \kappa_{1}^{2} \mu_{1}^{2}-\frac{1}{2} \kappa_{2}^{2} \mu_{2}^{2} .
\end{aligned}
$$

It should be noted that during obtaining the optimal fits to $\kappa_{i}$ and $\mu_{i}$, the variational theory is expected to set up a detailed fractional admixture of all the contributions coming from each single parameter $\left(U, \alpha, \Omega_{\varrho}\right.$ and $\left.\Omega_{z}\right)$ characterising the system.

We should remark that the oscillator-oscillator type waveform $(15,16)$ for $\phi(\mathbf{R}) \times \varphi(\mathbf{r})$ has proved to be the most efficient approximation yielding the largest lower bound on $\eta_{\mathrm{c}}$ among the possible sets of different combinations of Pekar, Coulomb and oscillator type trialwavefunctions tackled earlier by Verbist et al. [8] for the three- and two-dimensional bipolarons. We hope that the results derived here will provide us a revision of the phase stability of bipolarons within a broader context beyond the already existing literature concerning the bulk and strict $2 \mathrm{D}$ cases. We shall set up a total description of the problem uncovering the quasi-two-, -one- and -zerodimensional configurations as a function of the degree of confinement, and devote our concern mainly to the basic qualitative aspects of the confined bipolaron in the strong $\alpha$ limit. We shall not concern ourselves seriously with the numerals in absolute sense since it has been found that the value of $\eta_{\mathrm{c}}$ lays out a considerable amount of digression depending on the different types of wavefunctions used for the centre of mass and relative motions ( $c f$. Tab. 1 in Ref. [8]). We therefore disregard all the other possible forms for $\Phi(\mathbf{R}, \mathbf{r})$, and adopt plainly the oscillatoroscillator wavefunction as the most feasible approximation which, on the other hand, lends a great amount of computational simplification due to its compatibility with the quadratic barriers of the confining potential.

The explicit analytic form of the variational ground state energy $E_{\mathrm{g}}(11)$ is given through a series of closed but somewhat lengthy expressions which we prefer to present in Appendix A.

\section{Results and conclusions}

Since analytical minimization of $E_{\mathrm{g}}$ (A.1) is not possible, the determination of the optimal fits to the variational parameters $\left\{\kappa_{i}, \mu_{i}\right\},(i=1,2)$, requires treatment on a computer. In the foregoing discussions we shall be content with the limited information the strong coupling approximation can impart to the understanding of the problem, and devote our concern mainly to the qualitative aspects of the model uncovering different geometries and dimensionalities other than those for the 3D- and 2D-analogs which have already been investigated extensively $[6-9,12]$ within the framework of the same approximate adiabatic theory.

The fundamental condition under which a bipolaron can exist is that the repulsive Coulomb interaction should not be too strong to dominate over and hence break up the phonon-mediated binding which holds the particles together. It has been found that for a bipolaron state to be stable, one should have the ratio $U / \alpha$ be smaller than a critical value. For instance, in three dimensions, adopting the strong coupling theory and using oscillator-type wavefunctions $(15,16)$ for $\phi(\mathbf{R})$ and $\varphi(\mathbf{r})$, one obtains the bipolaron phase when $U / \alpha<1.627$. The corresponding critical bound in two dimensions is inevitably larger $(U / \alpha<1.679)$ due to the pronounced effective phonon coupling. The criterion for which a stable bipolaronic state takes place can be derived by demanding that the energy of the system of two interacting polarons be lower than twice that of a single polaron. Stating alternatively, for the bipolaron formation to be favourable, one requires

$$
E_{\mathrm{g}}-2 E_{\mathrm{g}}^{(1)}=\varepsilon<0
$$

where $E_{\mathrm{g}}^{(1)}$ refers to the one-polaron ground state energy, calculated within the identical framework as for the bipolaron system and under the identical numerical precision. The corresponding one polaron energy results can readily be recovered from reference [17]. For completeness, we review a brief content of this reference in Appendix B where the single electron wavefunction is provided by the same oscillator form as given by equation (15).

In conformity with the inequality $\varepsilon<0$, the condition which favours the bipolaron phase is that parameter $\eta(4)$ must be smaller than the critical value $\eta_{\mathrm{c}}=\left.\eta\right|_{\varepsilon=0}$ (i.e., the value at which $E_{\mathrm{g}}$ and $2 E_{\mathrm{g}}^{(1)}$ cross over). Clearly, the approximate $\eta_{\mathrm{c}}$ thus determined may lie either below or above its actual value depending on respectively whether the discrepancy between the variational and true energies for the bipolaron is larger or smaller than the corresponding discrepancy for two separated polarons. Although, at this point, we do not have a solid ground to judge or differentiate between the two possibilities, we are inclined to suppose that the variational error should be somewhat smaller for the one-polaron case. The underlying reason in that is the complexity of the situation for the bipolaron state where the intricate and opposing roles which the Coulomb and phonon parameters $(U, \alpha)$ play in the problem limits our skills in selecting an appropriate trial wavefunction that suits to give the least possible energy 


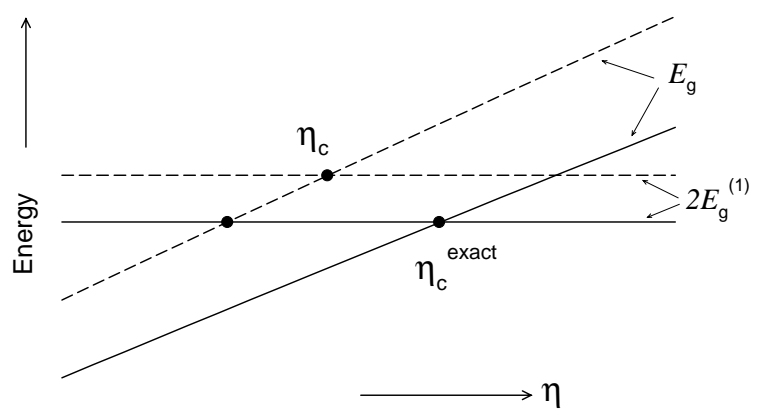

Fig. 1. $E_{\mathrm{g}}$ and $2 E_{\mathrm{g}}^{(1)}$ versus $\eta$. The full curves are the supposed exact energies, the dashed curves represent the corresponding variational upper bounds. The crossover point at which the full curves meet yield the presumably-exact value of $\eta_{\mathrm{c}}$. Similarly, the point at which the dashed lines cross gives the variationally computed $\eta_{\mathrm{c}}$. The crossover point on the very left refers to the critical $\eta$ determined by comparing the variational bipolaron energy with the exact single polaron energy.

upper bound. Apparently, the additional parameters $\Omega_{\varrho}$ and $\Omega_{z}$ add more to the complexity by greatly influencing the Coulomb and phonon counterparts of the problem and their percentual involvements in the binding (or dissociation). In view of this reasoning we feel that the variational estimate of $\eta_{\mathrm{c}}$ is probably smaller, rather than being larger, than its true value. Thus, adopting the assumption that $\eta_{\mathrm{c}}<\eta_{\mathrm{c}}^{\text {exact }}$, we think it would be somewhat more consistent to rely on the approach where both $E_{\mathrm{g}}$ and $E_{\mathrm{g}}^{(1)}$ are calculated variationally under similar approximations, rather than to have $\eta_{\mathrm{c}}$ determined by comparing the variational bipolaron energy with the exact single polaron energy derived from a superior formalism or any possible reference (see, for instance, Miyake [20], and $\mathrm{Wu}$ and Peeters [21] for the $3 \mathrm{D}$ and $2 \mathrm{D}$ cases). We provide a graphic summary of the content of our arguments in Figure 1 illustrating the pure-variational $\eta_{\mathrm{c}}$ to lie closer to the true value than the one calculated using the exact one-polaron results.

A complementary note concerning the variational adiabatic approach adopted in this problem is that it has a limited domain of applicability and can meet the more sensible and reliable approximations only in the limit of large $\alpha$. An intuitive envision at this stage would be to think of this theory as sufficiently valid for $\alpha$ being somewhat of the order of magnitude 10 or even larger in 3D and inclined to fall off to correspondingly smaller values as the degree of confinement is increased. To give a quantitative display of this trend we compare the ground state binding energy values of harmonically confined "one-polaron" states calculated within the academic strong-coupling approximation [19] against those derived using the superior path-integral formalism [22]. In Figure 2 we plot three successive domains in the $\alpha-\Omega$ space for the quasi-two-, -one- and -zero-dimensional geometries within which the strong-coupling one-polaron energies deviate from the corresponding path-integral results by at most $20 \%$. It is seen that the common fundamental effect of reducing the dimensionality and/or increasing the confining parameter $\Omega$

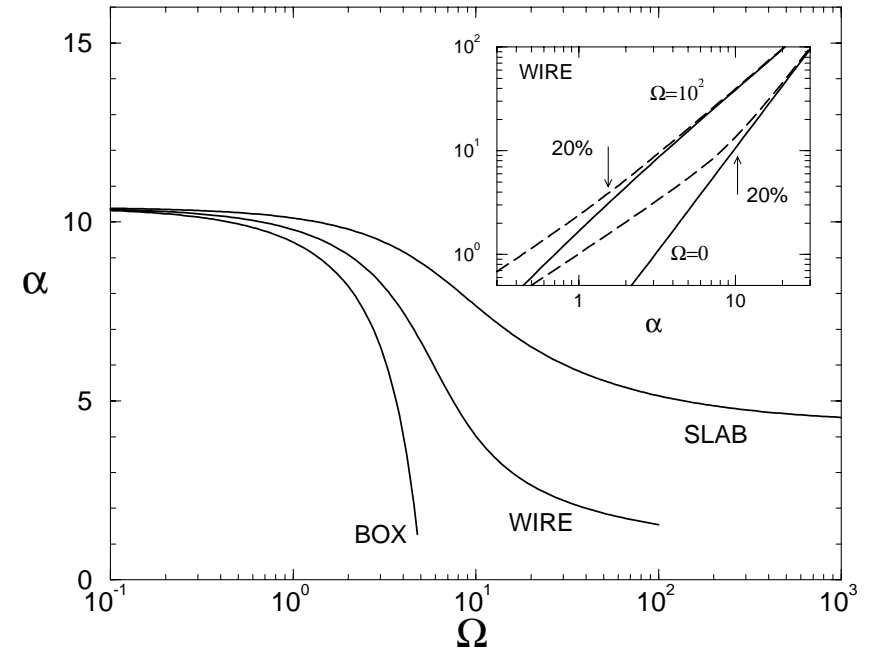

Fig. 2. Diagram describing the domains of applicability of the strong-coupling (SC) approximation where the one-polaron binding energy deviates from the corresponding path-integral (PI) value by at most $20 \%$. In the inset, the curves (solid: SC, dashed: PI) display the binding energy against $\alpha$ in the Q1Dconfiguration for $\Omega=0$ and $\Omega=10^{2}$. The vertical arrows refer to the corresponding $\alpha$-values for which the SC-theory lies deviated below the PI-theory by $20 \%$.

is to enlarge the domain of validity of the strong coupling approximation. Thus, based on a quantitative comparison of the strong-coupling and path-integral theories pertaining to the one-polaron case, we feel that the strong coupling approximation applied to the present problem will provide us with fairly acceptable values for $\alpha>10$ in 3D and $\alpha>4$ in 2D. Going over to the Q1D-confinement with $\Omega$ set equal to 40, for instance, the corresponding lower bound to $\alpha$ for which the adiabatic theory lies within $20 \%$ digression shifts down to a value as small as 2 . The overall content of Figure 2 is therefore to shed some crude insight into the effect of dimensionality and the degree of confinement concerning the validity of the approximation followed in this work.

In regard with the strong coupling expansion to leading order in $\alpha$, i.e., $\mathcal{O}\left(\alpha^{2}\right)$, we would like to draw attention to that, if in equations $(2,3)$ the energies are scaled by $\alpha^{2}$ and lengths by $\alpha$, i.e., $E \rightarrow E \alpha^{2}$ and $L \rightarrow L \alpha$, the only modification in the Hamiltonian would be to replace the confining parameter $\Omega$ by $\Omega / \alpha^{2}$ and the Coulomb coefficient $U$ by $U / \alpha$. Thus, in a representation where the critical value of parameter $\eta$ is plotted against $\Omega / \alpha^{2}$, rather than $\Omega$, we find that, irrespective of the value of $\alpha$, one can display the phase boundary on a single universal curve for each individual geometry. In every case, whatever the geometric configuration is, the ground state energy is seen to be proportional to the square of the coupling constant, i.e., $E_{\mathrm{g}}=-\mathcal{C} \alpha^{2}$, where the corresponding coefficient of proportionality bears a functional relation solely to $\Omega / \alpha^{2}$ for both the single-polaron and two-polaron systems. Therefore, in the foregoing particular plots for which the abscissae are expressed in units of the ratio $\Omega / \alpha^{2}$, one can 
Table 1. The ground state energy of the two-polaron complex compared with twice that of a single polaron in the quasi$2 \mathrm{D}$ configuration. The energy values are given in positive terms where the corresponding lowest subband energies have been subtracted out, i.e., $\Delta E_{\mathrm{g}}=\Omega-E_{\mathrm{g}}$ and $\Delta E_{\mathrm{g}}^{(1)}=\frac{1}{2} \Omega-E_{\mathrm{g}}^{(1)}$. Those entries typed in boldface correspond to the set of parameter values, $\alpha, \eta$ and $\Omega$, for which bipolaron formation is favourable.

\begin{tabular}{|c|c|ccccccc|}
\hline$\Omega / \alpha^{2}$ & $2 \Delta E_{\mathrm{g}}^{(1)} / \alpha^{2}$ & \multicolumn{7}{|c|}{$\Delta E_{\mathrm{g}} / \alpha^{2}$} \\
\cline { 3 - 8 } & & $\eta=0.01$ & $\eta=0.10$ & $\eta=0.12$ & $\eta=0.13$ & $\eta=0.14$ & $\eta=0.15$ & $\eta=0.16$ \\
\hline \hline 0 & 0.2122 & $\mathbf{0 . 2 5 5 8}$ & $\mathbf{0 . 2 2 4 1}$ & $\mathbf{0 . 2 1 6 6}$ & $\mathbf{0 . 2 1 2 7}$ & 0.2089 & 0.2050 & 0.2010 \\
\hline $10^{-2}$ & 0.2219 & $\mathbf{0 . 2 6 5 4}$ & $\mathbf{0 . 2 3 3 7}$ & $\mathbf{0 . 2 2 6 2}$ & $\mathbf{0 . 2 2 2 3}$ & 0.2184 & 0.2145 & 0.2105 \\
\hline $10^{-1}$ & 0.2830 & $\mathbf{0 . 3 2 7 0}$ & $\mathbf{0 . 2 9 2 4}$ & $\mathbf{0 . 2 8 4 1}$ & 0.2799 & 0.2756 & 0.2713 & 0.2669 \\
\hline 1 & 0.4362 & $\mathbf{0 . 4 9 0 5}$ & $\mathbf{0 . 4 4 2 6}$ & 0.4311 & 0.4252 & 0.4193 & 0.4133 & 0.4072 \\
\hline 10 & 0.6018 & $\mathbf{0 . 6 8 4 0}$ & $\mathbf{0 . 6 2 0 2}$ & $\mathbf{0 . 6 0 4 9}$ & 0.5971 & 0.5891 & 0.5811 & 0.5729 \\
\hline $10^{2}$ & 0.7100 & $\mathbf{0 . 8 2 4 8}$ & $\mathbf{0 . 7 4 8 9}$ & $\mathbf{0 . 7 3 0 7}$ & $\mathbf{0 . 7 2 1 3}$ & $\mathbf{0 . 7 1 1 9}$ & 0.7022 & 0.6924 \\
\hline $10^{3}$ & 0.7589 & $\mathbf{0 . 8 9 3 9}$ & $\mathbf{0 . 8 1 1 8}$ & $\mathbf{0 . 7 9 1 9}$ & $\mathbf{0 . 7 8 1 8}$ & $\mathbf{0 . 7 7 1 5}$ & $\mathbf{0 . 7 6 1 1}$ & 0.7505 \\
\hline$\infty$ & 0.7854 & $\mathbf{0 . 9 3 3 3}$ & $\mathbf{0 . 8 4 7 3}$ & $\mathbf{0 . 8 2 6 6}$ & $\mathbf{0 . 8 1 6 0}$ & $\mathbf{0 . 8 0 5 2}$ & $\mathbf{0 . 7 9 4 3}$ & 0.7832 \\
\hline
\end{tabular}

conveniently assign $\alpha$ any arbitrary large value with no loss in generality.

In Table 1 we give an explicit numerical discourse of the binding energy values of the "two-polaron" system compared with that of infinitely separated noninteracting polarons. We shall be content by tabulating a sample of our numerical results pertaining to only the slab-like configuration where we set $\Omega_{\varrho}=0$ and select a succession of $\Omega_{z}$ values ranging between infinity and zero, thus encompassing the integer dimensional space limits which are of immediate relevance to the three- and two-dimensional one- and two-polaron models studied in the literature in so far. We should recall that for the case of a free single polaron the binding energy calculated by the oscillator-type wavefunction is given by

$$
-\frac{E_{\mathrm{g}}^{(1)}}{\alpha^{2}} \equiv \mathcal{C}\left(\frac{\Omega}{\alpha^{2}}\right)=\left\{\begin{array}{ll}
(3 \pi)^{-1} & \text { for } \Omega=0 \\
\pi / 8 & \text { for } \Omega \rightarrow \infty
\end{array} .\right.
$$

The more general values of the coefficient $\mathcal{C}\left(\Omega / \alpha^{2}\right)$, extended to non-integer dimensionality $(0 \leq \Omega<\infty)$, are displayed in a succession of columns in the table for the one- and two-polaron cases with a series of distinctive Coulomb repulsion strengths; thus yielding an explicit tracking of the condition, equation (18), for which a bipolaronic bound state is favourable.

In Figure 3 we plot three simultaneous curves describing the effect of confinement on the critical value of $\eta$ in the quasi-two, -one and -zero dimensional configurations. Holding $\alpha$ fixed at any desired value and following the variations in $\eta_{\mathrm{c}}$ as $\Omega$ is turned on, we first note that for each dimensionality the critical $\eta$, starting from the common $3 \mathrm{D}$-value, $\eta_{\mathrm{c}}^{(3 \mathrm{D})}=0.131$, displays in common a decreasing trend where the respective decay rates are observed to become faster as the effective dimensionality is reduced from two to one, and to zero. Thus, in the wire- and box-type configurations the critical Coulomb strength below which a bipolaron forms lies deviated considerably below the corresponding value in the slab-type geometry. The essential feature portrayed by the strong coupling theory is therefore that, the lower the dimensionality is, the more unlikely is to realize the

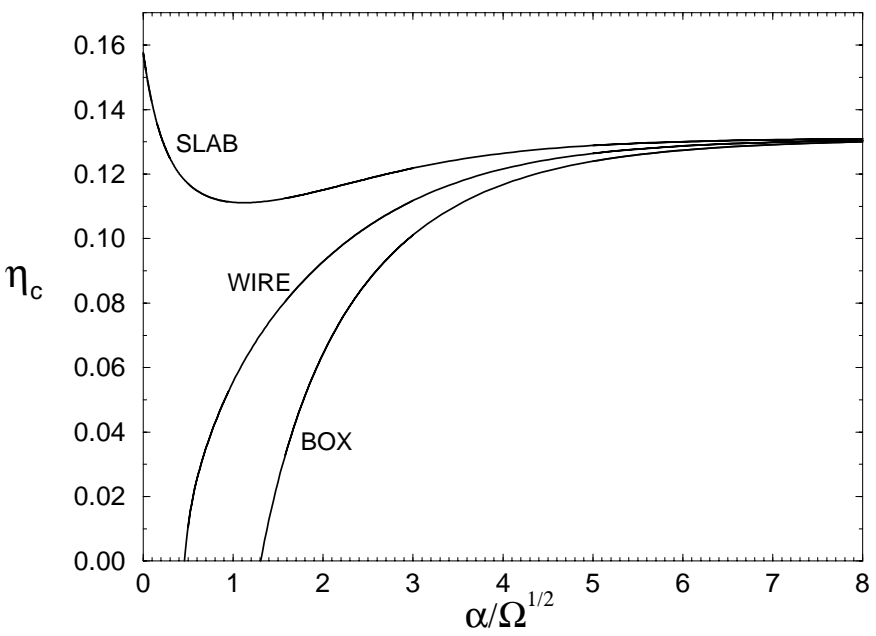

Fig. 3. The critical ratio $\eta_{\mathrm{c}}$ as a function of $\alpha / \sqrt{\Omega}$ for the quasi-two-, -one- and -zero-dimensional configurations.

bipolaron state to form; and in particular, in the box-type confinement, even a small value of $\eta$ may turn out to be sufficient enough to lead the Coulomb force to oppose and dominate over the phonon-coupling-induced localisation of the composite assembly of the two nearby electrons. The same feature has also been depicted explicitly in an earlier paper by Mukhopadhyay and Chatterjee [14] for bipolarons in quantum dots. As an immediate reasoning to understand such a characteristic of low-dimensional bipolarons treated within the adiabatic theory we are tempted to argue that, with increasing degree of confinement (i.e., with growing $\Omega_{\varrho}$ and/or $\Omega_{z}$ ), the Coulomb repulsion is steadily strengthened due to that the particles are squeezed to get closer; and the rate at which this happens should be most prominent in the Q0Dconfiguration where the electrons are pushed towards one another from all radially inward directions. In the Q1Dand Q2D-configurations, however, the electrons are free to expand and relax themselves respectively in either one or two directions; thus resulting with comparatively weaker 
Coulomb repulsion and weaker dominating strength over the lattice polarization field holding the particles together.

In contrast with the outcomes of the present work and those of reference [14], we should mention two parallel contravening papers by Wan et al. [15] and by Pokatilov et al. [16], both treating the Q0D-bipolaron problem within the framework of the path-integral formulation and leading to the common finding that, unlike to the strong-coupling results, the confinement effects act in favour of bipolaronic stability in the zero-dimensional configuration. Moreover, it has been argued that, even at weak coupling, bipolarons can form in quantum dots under strong confinement [15]. Since the path-integral formalism is highly superior over the strong-coupling approximation, we think that the counter-view laid out in Figure 3 regarding the box-geometry is rather suspect and should be accounted for as an artifact of the adiabatic theory. Thus, having pointed out this particular conflicting digression between the two theories, we continue with our discussions within the present context to lay out a complete and selfconsistent characterisation of strong-coupling bipolarons in confined media uncovering a broad variety of the basic features induced by the adiabatic theory.

We should note that in confined systems it is not only the Coulomb repulsion strength which gets pronounced, but in the meantime the phonon-coupling becomes pseudo-enhanced leading to a more effective and deeper polaronic binding to oppose and counterbalance the repulsive forces. The competitive interrelation between these aspects of the problem determines the phase boundary and moreover may even pose a salient feature stemming from a cross-overing of this competition as the confining parameters are varied. In particular, in the Q2D slab-geometry where this feature shows up with extreme prominence, we observe that the critical $\eta$ does not display a monotonically increasing behavior interpolating between the 3D- and 2D-limits, but instead reaches its twodimensional value $[8]\left(\eta_{\mathrm{c}}^{(2 \mathrm{D})}=0.158\right)$ after having passed through a minimum; thus reflecting an explicit image of the dominating effect of either the Coulomb repulsion or the phonon mediated attraction over the other.

Peculiar to the slab geometry, we note explicitly that, whatever the value of $\Omega$ is, there is always a non-zero critical $\eta$ below which a stable bipolaronic state can be realized. In the wire- and box-type confinements, however, no matter how weak the Coulomb strength might be set, there is always an upper value for $\Omega$ beyond which the bipolaron breaks up into two individual polarons. Increased values of $\alpha$ can only support the bipolaron to conserve its stability at correspondingly higher degrees of confinement. A more clear picture of such a trait pertaining to the quasi-two-, -one- and -zero-dimensional geometries is provided by the phase diagrams in Figure 4 where we plot the Coulomb coefficient $U$ against $\alpha$. Scaling both the abscissa and the ordinate in units of $\Omega^{1 / 2}$ allows us to display the stability region for any desired $\Omega$-value. In the phase picture the space lying below the dashed line corresponds to $\eta_{\mathrm{c}}<0$, and is therefore unphysical. The upper space bounded from below by the solid lines plotted for

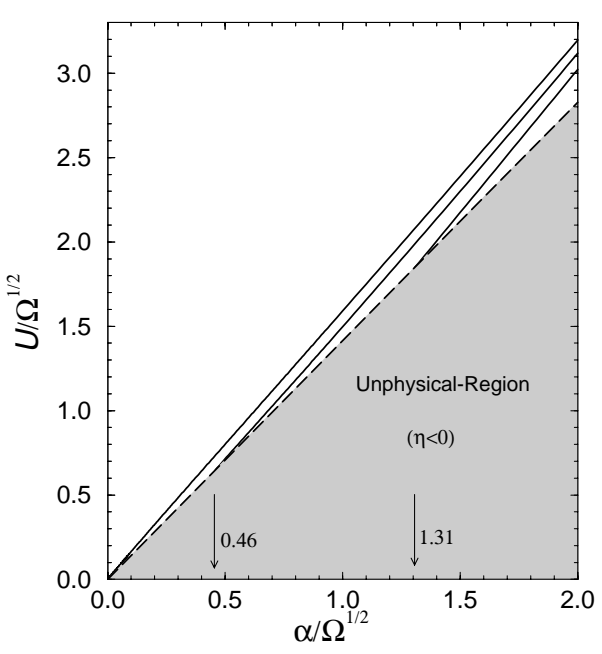

Fig. 4. The phase diagram for bipolaron formation as a function of the Coulomb coefficient and electron-phonon coupling constant. The solid lines from top to bottom are respectively, for the quasi-two-, -one- and -zero-dimensional configurations. The dashed line $\left(\eta_{\mathrm{c}}=0\right)$ is the boundary of the unphysical region. The arrows refer to the corresponding critical values of $\alpha$ (in units of $\Omega^{1 / 2}$ ) in the wire- and box-type confinements.

different geometries gives the unstable region where the polarons choose to remain separated. Thus, it is only the narrow triangular area bounded by the dashed and either of the solid lines in which the polarons can be found in a bound state forming a stable bipolaron. The vertex of each triangular region at which the dashed and solid lines join defines an infimum for the coupling constant for a given $\Omega$-value. We observe that the critical $\alpha$ below which the unphysical region is obtained gets shifted to larger values as the degree of confinement is increased. It should be remarked that in the Q2D-configuration the solid and dashed lines join at the origin; yielding an evidence in favour of bipolaronic stability for any degree of confinement provided the Coulomb coefficient does not exceed its corresponding critical value.

In this article we have studied the possibility and criteria in achieving stable bipolarons in low dimensionally confined media. The "deformable potential box" - model adopted in this work allows us to attain a simple and yet comprehensive review of the two-polaron problem within a unifying scheme interpolating between the bulk and all low dimensional geometric configurations of general interest. It has been illustrated that in structures with reduced dimensionality, the phase description displays an explicit relevance to the phonon-coupling parameter $\alpha$, distinguished from that reported earlier for the strongly coupled bipolaron in three and two dimensions. It has been shown further that in conforming the potential box to a thin quantum well, the critical ratio of the dielectric constants may undergo an interesting variation, exhibiting a decrease first, then ascending and eventually going over to its topmost two-dimensional value. 
R.T. Senger acknowledges the Ph.D. Scholarship offered by the Münir Birsel Foundation - TÜBİTAK.

\section{Appendix A: Variational bipolaron energy}

In complete form, the ground state energy (11) of the composite system of two polarons is evaluated as

$$
E_{\mathrm{g}}=\lambda_{R}+\lambda_{r}+\Omega_{\varrho}^{2} \lambda_{\varrho}+\Omega_{z}^{2} \lambda_{z}+\lambda_{\mathrm{c}}-\lambda_{\mathrm{ph}} .
$$

In the above, $\lambda_{R}$ and $\lambda_{r}$ stand for the kinetic terms $\left\langle\frac{1}{4} P^{2}\right\rangle$ and $\left\langle p^{2}\right\rangle$, respectively; $\lambda_{\varrho}$ and $\lambda_{z}$ are the energies relevant to the confining potential; $\lambda_{\mathrm{c}}$ is the Coulomb energy and $\lambda_{\text {ph }}$ refers to the contribution coming from the lattice. We obtain the following explicit expressions for each of these terms:

$$
\begin{gathered}
\lambda_{R}=\left\langle-\frac{1}{4} \nabla_{R}^{2}\right\rangle_{\phi}=\frac{1}{8} \kappa_{1}^{2}\left(2+\mu_{1}^{2}\right) \\
\lambda_{r}=\left\langle-\nabla_{r}^{2}\right\rangle_{\varphi}=\kappa_{2}^{2}\left[\frac{2+3 \mu_{2}^{2}+2 \mu_{2}^{4}}{2+4 \mu_{2}^{2}}\right] \\
\lambda_{\varrho}=\left\langle R_{\varrho}^{2}\right\rangle_{\phi}+\left\langle\frac{1}{4} r_{\varrho}^{2}\right\rangle_{\varphi}=\frac{1}{\kappa_{1}^{2}}+\frac{1+4 \mu_{2}^{2}}{4 \kappa_{2}^{2}\left(1+2 \mu_{2}^{2}\right)} \\
\lambda_{z}=\left\langle R_{z}^{2}\right\rangle_{\phi}+\left\langle\frac{1}{4} r_{z}^{2}\right\rangle_{\varphi}=\frac{1}{2 \kappa_{1}^{2} \mu_{1}^{2}}+\frac{3+2 \mu_{2}^{2}}{8 \kappa_{2}^{2} \mu_{2}^{2}\left(1+2 \mu_{2}^{2}\right)} .
\end{gathered}
$$

Defining, for notational convenience,

$$
F_{n}(t) \equiv \int_{0}^{\infty} \mathrm{d} x x^{n} \mathrm{e}^{(1-t) x^{2}} \operatorname{erfc}(x)
$$

with erfc denoting the complementary error function, we write

$$
\lambda_{\mathrm{c}}=\left\langle U r^{-1}\right\rangle_{\varphi}=2 U \frac{\mu_{2} \kappa_{2}}{1+2 \mu_{2}^{2}}\left\{\frac{1}{\sqrt{\pi}}+\mu_{2} F_{0}\left(\mu_{2}^{2}\right)\right\} .
$$

Also, calculating $s_{Q}(10)$; and using equation (A.6), we derive

$$
\begin{aligned}
\lambda_{\mathrm{ph}}= & \sum_{Q} V_{Q}^{2} s_{Q}^{2}=\frac{\alpha \sqrt{2}}{\pi} \int_{0}^{\infty} \mathrm{d} q q \int_{0}^{\infty} \mathrm{d} q_{z} \frac{s_{Q}^{2}}{q^{2}+q_{z}^{2}} \\
= & 2 \sqrt{2} \alpha b\left\{F_{0}(\xi)+\delta\left(\frac{b}{2}\right)^{2} F_{2}(\xi)+\frac{\delta^{2}}{2}\left(\frac{b}{2}\right)^{4} F_{4}(\xi)\right\} \\
& -\frac{\alpha a^{2} b \delta}{2 \sqrt{2 \pi}\left(1-\mu_{2}^{4}\right)}\left\{1+\frac{a^{2} \delta}{2^{4}\left(1-\mu_{2}^{4}\right)}\left[1-\frac{1}{2} \xi-2 \mu_{2}^{4}\right]\right\}
\end{aligned}
$$

in which

$$
a=\left\{\frac{8 \kappa_{1}^{2} \kappa_{2}^{2}}{\kappa_{1}^{2}+4 \kappa_{2}^{2}}\right\}^{1 / 2}, \quad b=\left\{\frac{8 \kappa_{1}^{2} \kappa_{2}^{2} \mu_{1}^{2} \mu_{2}^{2}}{\mu_{1}^{2} \kappa_{1}^{2}+4 \mu_{2}^{2} \kappa_{2}^{2}}\right\}^{1 / 2}
$$

and

$$
\xi=\frac{b^{2}}{a^{2}}, \quad \delta=\frac{\mu_{2}^{-2}-\mu_{2}^{2}}{\kappa_{2}^{2}\left(1+2 \mu_{2}^{2}\right)}
$$

\section{Appendix B: Variational one-polaron energy}

In the one polaron problem the Hamiltonian $(2,3)$ reduces to

$$
\begin{aligned}
\mathrm{H}= & \frac{1}{2} p^{2}+\frac{1}{2}\left(\Omega_{\varrho}^{2} \varrho^{2}+\Omega_{z}^{2} z^{2}\right) \\
& +\sum_{Q} a_{Q}^{\dagger} a_{Q}+\sum_{Q} V_{Q}\left(a_{Q} \mathrm{e}^{\mathrm{i} \mathbf{Q} \cdot \mathbf{r}}+\mathrm{hc}\right)
\end{aligned}
$$

where $\mathbf{r}=(\varrho, z)$ is the electron position, and $\mathbf{p}$ is its momentum.

Using the same variational approximation as for the bipolaron case, we impose a product Ansatz separable in the particle and phonon coordinates, i.e.,

$$
\Psi^{(1)}=\phi^{(1)}(\varrho, z) \exp \sum_{Q} s_{Q}\left(a_{Q}-a_{Q}^{\dagger}\right)|0\rangle
$$

in which we use an oscillator particle-wavefunction

$$
\phi^{(1)}(\varrho, z)=N \exp \left\{-\frac{1}{2} \kappa^{2}\left(\varrho^{2}+\mu^{2} z^{2}\right)\right\} .
$$

The exponential operator acting on the phonon vacuum is the displaced oscillator transformation where $s_{Q}$ is a variational parameter determined from the requirement: $\partial\left\langle\Psi^{(1)}|\mathrm{H}| \Psi^{(1)}\right\rangle / \partial s_{Q}=0$ to yield

$$
\begin{aligned}
s_{Q} & =V_{Q}\left\langle\phi^{(1)}\left|\mathrm{e}^{ \pm \mathbf{i} \mathbf{Q} \cdot \mathbf{r}}\right| \phi^{(1)}\right\rangle \\
& =\exp \left(-\frac{1}{2 \kappa^{2} \mu^{2}}\left(\mu^{2} q^{2}+q_{z}^{2}\right)\right) .
\end{aligned}
$$

Finally, using (B.4) in (B.2) and projecting out the Qsummations in $E_{\mathrm{g}}^{(1)}=\left\langle\Psi^{(1)}|\mathrm{H}| \Psi^{(1)}\right\rangle$, we obtain the onepolaron ground state energy, given by

$$
\begin{aligned}
E_{\mathrm{g}}^{(1)}= & \frac{1}{4} \kappa^{2}\left(2+\mu^{2}\right)+\frac{1}{2 \kappa^{2}}\left(\Omega_{\varrho}^{2}+\frac{\Omega_{z}^{2}}{2 \mu^{2}}\right) \\
& -\frac{\alpha}{\sqrt{\pi}} \kappa \mu \frac{\arctan \sqrt{\mu^{2}-1}}{\sqrt{\mu^{2}-1}} .
\end{aligned}
$$

\section{References}

1. Y. Takada, Phys. Rev. B 26, 1223 (1982).

2. H. Hiramoto, Y. Toyozawa, J. Phys. Soc. Jpn 54, 245 (1985).

3. J. Adamowski, Phys. Rev. B 39, 3649 (1989).

4. T.K. Mitra, Phys. Lett. A 142, 398 (1989).

5. F. Bassani, M. Geddo, G. Iadonisi, D. Ninno, Phys. Rev. B 43, 5296 (1991).

6. G. Verbist, F.M. Peeters, J.T. Devreese, Phys. Rev. B 43, 2712 (1991).

7. S. Sil, A.K. Giri, A. Chatterjee, Phys. Rev. B 43, 12642 (1991). 
8. G. Verbist, M.A. Smondyrev, F.M. Peeters, J.T. Devreese, Phys. Rev. B 45, 5262 (1992).

9. C. Qinghu, W. Kelin, W. Shaolong, Phys. Rev. B 50, 164 (1994).

10. J. Adamowski, S. Bednarek, J. Phys. Cond. Matter 4, 2845 (1992).

11. A. Chatterjee, S. Sil, Int. J. Mod. Phys. B 7, 4763 (1993).

12. F. Luczak, F. Brosens, J.T. Devreese, Phys. Rev. B 52, 12743 (1995).

13. S. Sahoo, J. Phys. Cond. Matter 7, 4457 (1995).

14. S. Mukhopadhyay, A. Chatterjee, J. Phys. Cond. Matter 8, 4017 (1996).

15. Y. Wan, G. Ortiz, P. Phillips, Phys. Rev. B 55, 5313 (1997).
16. E.P. Pokatilov, V.M. Fomin, J.T. Devreese, S.N. Balaban, S.N. Klimin, J. Phys. Cond. Matter 11, 9033 (1999).

17. D.M. Larsen, in Proc. 17th Int. Conf. on the Physics of Semiconductors, edited by J.D. Chadi, W. Harrison (Berlin, Springer, 1985), p. 421.

18. S.N. Klimin, E.P. Pokatilov, V.M. Fomin, J. Phys. Status Solidi (b) 184, 373 (1994).

19. T. Yıldırım, A. Erçelebi, J. Phys. Cond. Matter 3, 1271 (1991).

20. S.J. Miyake, J. Phys. Soc. Jpn 38, 181 (1975).

21. X.G. Wu, F.M. Peeters, Phys. Rev. B 31, 3420 (1985).

22. R.T. Senger, A. Erçelebi, J. Phys. Cond. Matter 9, 5067 (1997). 